\title{
Accelerating restrictive cardiomyopathy after liver transplantation in a patient with familial amyloidotic polyneuropathy: a case report Jason Robin*1, Sheridan Meyers ${ }^{1}$, Maher Nahlawi ${ }^{1}$, Jyothy Puthumana ${ }^{1}$, Jon Lomasney2 ${ }^{2}$ David Mehlman ${ }^{1}$, Vera Rigolin ${ }^{1}$ and Charles Davidson ${ }^{1}$
}

Address: ${ }^{1}$ Department of Medicine, Division of Cardiology, Northwestern University Feinberg School of Medicine, Chicago, Illinois, USA and 2Department of Pathology, Northwestern University Feinberg School of Medicine, Chicago, Illinois, USA

Email: Jason Robin* - j-robin@md.northwestern.edu; Sheridan Meyers - snm@northwestern.edu; Maher Nahlawi - maher99@sbcglobal.net; Jyothy Puthumana - jputhumana@nmff.org; Jon Lomasney - j-lomasney@northwestern.edu; David Mehlman - d-mehlman@northwestern.edu; Vera Rigolin - v-rigolin@northwestern.edu; Charles Davidson - cdavidso@nmh.org

* Corresponding author

Published: I February 2008

Journal of Medical Case Reports 2008, 2:35 doi:10.1 186/1752-1947-2-35

Received: 15 August 2007

Accepted: I February 2008

This article is available from: http://www.jmedicalcasereports.com/content/2/1/35

(c) 2008 Robin et al; licensee BioMed Central Ltd.

This is an Open Access article distributed under the terms of the Creative Commons Attribution License (http://creativecommons.org/licenses/by/2.0), which permits unrestricted use, distribution, and reproduction in any medium, provided the original work is properly cited.

\begin{abstract}
Introduction: Hereditary amyloidodis is a rare disease process with a propensity to cause polyneuropathies, autonomic dysfunction, and restrictive cardiomyopathy. It is transmitted in an autosomal dominant manner, with disease onset usually in the 20s-40s. The most common hereditary amyloidogenic protein, transthyretin, is synthesized in the liver and lies on Chromosome 18. Over 80 amyloidogenic transthyretin mutations have been described, the majority of which are neuropathic and hence the common name, Familial Amyloidotic Polyneuropathy. Until 1990, the disease was intractable with a 5-15 year survival after diagnosis. The prognosis changed after the implementation of orthotropic liver transplantation as a treatment strategy which halts the synthesis of amyloidogenic transthyretin. This has now has been performed over 1300 times in 67 centers.
\end{abstract}

Case presentation: We describe the case of a man of Irish ancestry with Familial Amyloidotic Polyneuropathy and no clinical history of cardiac involvement. Shortly after orthotropic liver transplantation, he developed congestive heart failure. He was subsequently diagnosed with an accelerating post-transplant restrictive cardiomyopathy due to amyloid infiltration.

Conclusion: A liver transplant induced cardiomyopathy in Familial Amyloidotic Polyneuropathy can be observed in patients without any history of cardiac symptoms. All patients with Familial Amyloidotic Polyneuropathy should be followed after transplantation to assess for a deterioration in cardiac function.

\section{Introduction}

Familial Amyloid Polyneuropathy (FAP) is a disease process that has been described to affect the cardiovascular system. Clinical manifestations include hypotension, conduction disturbances, and most problematic, restrictive cardiomyopathies [1]. The majority of those with FAP have bothersome neuropathies, and are spared significant cardiovascular complications. Orthotropic liver transplantation (OLT) has been shown to stabilize and at times improve the neuropathic symptoms. Based on the pathogenesis of FAP, if OLT is performed prior to any of the clinical manifestations of cardiac amyloidosis, the likeli- 
hood of a patient succumbing to an amyloid cardiomyopathy should be significantly decreased.

\section{Case presentation}

A 61 year old man presented with increasing dyspnea on exertion, ascites, and lower extremity edema. His medical history was remarkable for FAP which initially manifested ten years earlier as nausea and vomiting due to gastroparesis. In addition, he developed a painful peripheral neuropathy approximately two years later. Due to his progressive symptoms, he underwent OLT at another institution in August, 2006. Prior to his transplant, he had no cardiovascular complaints. His preoperative evaluation included a 2-dimensional echocardiogram. He did not have a cardiac catheterization. Approximately 2 months after his transplant, he began feeling dyspneic with mild to moderate activity. Shortly thereafter, he began to develop increasing abdominal girth and lower extremity edema. He presented to our institution in February, 2007 with further progression of these symptoms. His current medications included prednisone, tacrolimus, gabapentin, clotrimazole, valcyclovir, and pentamadine. His family history was significant for a father dying in his 50s due to gastrointestinal complications from FAP. On physical examination his heart rate was 100 with a blood pressure of 110/60 mmHg. He had an oxygen saturation of $97 \%$ while receiving oxygen at $4 \mathrm{~L} / \mathrm{min}$ by nasal canula. He had no evidence of jaundice. He had crackles at the bases of his lungs bilaterally. His cardiovascular exam was remarkable for $10 \mathrm{~cm}$ of jugular venous distension, a regular rhythm with no murmurs, and an S4 gallop at the apex. His abdomen was mildly distended with shifting dullness to percussion and a liver edge $4 \mathrm{~cm}$ below the right costal margin. He had $2+$ bilateral lower extremity edema. The ECG demonstrated sinus tachycardia with normal voltage, right axis deviation, and a left posterior divisional block. The Chest XRAY demonstrated mild cardiomegaly, patchy infiltration with associated atelectasis at the lung bases and a small right sided pleural effusion. The echocardiogram demonstrated an increase in left atrial volume, but was otherwise unchanged from his preoperative echocardiogram. (Table 1) This prompted an inferiorvenocavogram to evaluate for possible stenosis at the level of the anastomosis. The pressure gradient across the inferior vena cava anastomosis was unremarkable at 1-2 mmHg. There was no evidence of portal hypertension. However, the overall central venous pressure was elevated at $18-19 \mathrm{mmHg}$. It was decided at this time to perform a right and left heart catheterization. The coronary arteries were angiographically normal. The right and left heart pressures were elevated. (Table 2) His pressure tracings demonstrated no evidence of intrathoracic-intracardiac pressure dissociation or ventricular interdependence. Overall, this pattern was most consistent with restrictive physiology. An endomyocardial biopsy was performed which was consistent with cardiac amyloidosis. (Figure 1) Subsequently, it was learned that the patient's mutation was on the transthyretin (TTR) gene, Threonine60Alanine. He was started on an aggressive diuretic regimen and began to feel moderate relief. He is currently being closely followed as an out-patient and will be considered for cardiac transplantation if his symptoms cannot be controlled with medical management.

\section{Discussion}

Amyloidosis is a disorder resulting from the abnormal deposition of a particular protein in various tissues of the body. The four most common forms of amyloidosis are: (1) light chain due to immunoglobulins; (2) secondary which is seen in chronic inflammatory states such as rheumatoid arthritis; (3) senile which is typically seen in those over the age of 80; and (4) heriditary. There are at least eight different proteins that have been recognized to cause the hereditary amyloidoses [2]. Of these, the amyloidgenicTTR (ATTR) protein is the most common, with the Val30Met (Portuguese type) being the most prevalent mutation causing ATTR ( $80 \%$ of cases) [2]. The most common manifestation of ATTR is a neuropathy, but clinical manifestations vary depending on the location of the mutation. The treatment of hereditary amyloidosis is OLT,

Table I: Echocardiograms: Pre- and Post- Liver Transplant

\begin{tabular}{lcc}
\hline Variable & May, 2006 & February, 2007 \\
\hline Ejection Fraction & $55 \%$ & $50 \%$ \\
Interventricular Septal Thickness (cm)* & 1.5 & 1.2 \\
Posterior Wall Thickness (cm)* & 1.2 & Moderate \\
Left Ventricular Hypertrophy & Moderate & 40 \\
Left Atrial Volume Index (cclm2)** & 24 & Grade 2 \\
Diastolic Dysfunction & Grade 2 & 35 \\
Right Ventricular Systolic Pressure (mmHg) & 25 & No \\
>mild valvular regurgitation & No & Trivial to Small \\
Pericardial effusion & No &
\end{tabular}

*Normal Value $0.6-1.1 \mathrm{~cm}$

**Normal Value $<28 \mathrm{cc} / \mathrm{m} 2$ 
Table 2: Right Heart Catheterization Pressures

\begin{tabular}{llllll}
\hline RA & RV & PA & PCWP & LV & AO \\
\hline 16 & $65 / 9$ & $63 / 32$ & 37 & $155 / 9$ & $154 / 82$
\end{tabular}

$-\mathrm{RA}=$ Right Atrium, RV = Right Ventricle, $\mathrm{PA}=$ Pulmonary Artery, $\mathrm{PCWP}=$ Pulmonary Wedge Pressure, $\mathrm{LV}=$ left Ventricle, $\mathrm{AO}=$ Aortic Pressure

-Pressures are recorded in $\mathrm{mmHg}$

-Normal Mean Values: RA ( $3 \mathrm{~mm} \mathrm{Hg})$, RV $(25 / 4 \mathrm{~mm} \mathrm{Hg})$, PA (25/9

$\mathrm{mm} \mathrm{Hg})$ PCWP $(9 \mathrm{~mm} \mathrm{Hg})$, LV (I30/8 mm Hg), AO (130/70 mm Hg)

which limits further synthesis of the mutated protein, and thus halts further deposition in the organs. The Val30Met mutation typically presents with neuropathy, cardiac conduction disturbances, GI dysfunction, nephrosis and carpal tunnel syndrome. The eighty-plus nonVal30Met mutations in general have a greater propensity to cause cardiomyopathies [1]. In instances of a concomitant cardiomyopathy in the setting of FAP, simultaneous heartliver transplantation has been performed with some success, though the numbers are small $[3,4]$.

What we describe here is a man with no clinical manifestations of a cardiomyopathy prior to OLT who developed a progressive cardiomyopathy shortly after obtaining a normal liver. This clinical presentation of rapid and progressive cardiac amyloidosis following liver transplantation for FAP has been described by Stangou et al. His group evaluated 20 patients with FAP, 14 of whom under-

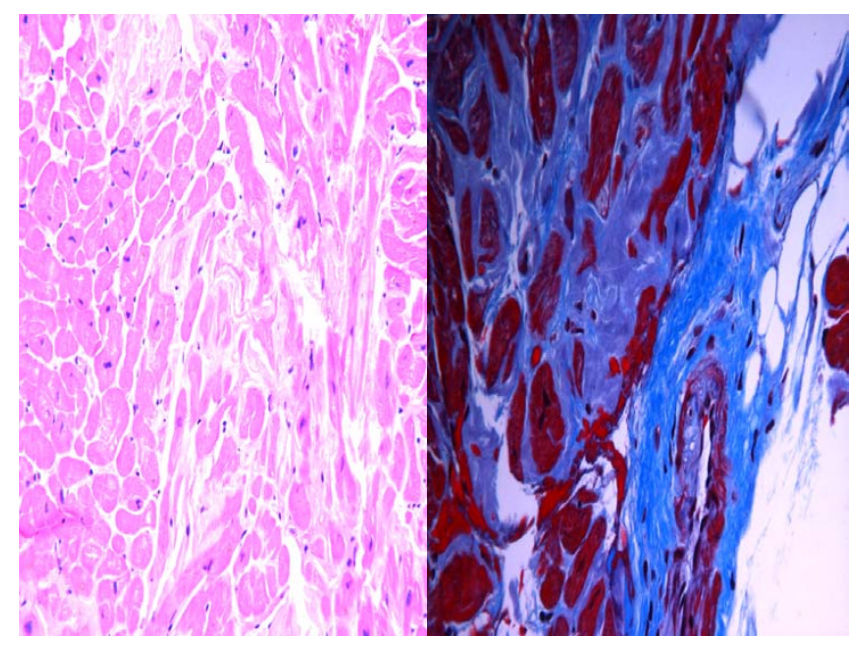

\section{Figure I}

a. (Left) H\&E. Higher power shot. Eosinophilic amorphous material separating myocytes with angulation of myocytes consistent with amyloidosis. b. (Right) Trichrome stain. Collagen around blood vessel (lower right corner) is blue, material surrounding myocytes is grey characteristic of amyloid. Interstitial material exhibited apple-green birefringence under polarized light with Congo red staining. (Not shown) went OLT, and compared their post-operative cardiac course with 10 traditional cirrhotic patients who underwent OLT. He found that in the group of FAP patients with OLT, there was a significant change in mean interventricular septal thickness in the 5 patients with nonVal30met mutations $(15.2 \mathrm{~mm}$ progressed to 21.5 $\mathrm{mm}$ over 3 months, $\mathrm{p}<0.05)$. There was no significant change in those with Val30Met mutations who underwent OLT, those with FAP who did not receive a liver or in the patients with traditional cirrhosis after OLT. In addition, 2 of the nonVal30Met patients were dead at 3 months due to heart failure [5]. Of interest, Olofsson et al also demonstrated progressive ventricular thickness in a group of FAP patients post-OLT with the more common Val30Met mutation, though the degree of ventricular hypertrophy was not as dramatic as was seen in Stangou's populatin (mean IVS thickness $11.5 \mathrm{~mm}$ before OLT and $13.1 \mathrm{~mm}$ at 18 months) [6].

The current hypothesis as to why this may occur has been looked at by previous investigators. Biochemical evidence suggests that before OLT, variant TTR-derived amyloid fibrils form a template to which wild type TTR attaches to after OLT. Yazaki et al studied 6 patients with FAP and evidence of cardiac involvement. Amyloid fibrils of the heart were composed of wild-type TTR as well as variant TTR at a ratio of about $1: 1$ in 5 patients without liver transplantation. In the patient with a transplanted liver, about $80 \%$ of the cardiac amyloid consisted of wild-type TTR [7]. He concluded that wild-type TTR contributes greatly to the development of amyloid deposition in the heart of FAP patients after OLT. This hypothesis is still considered by most experts to be the most likely explanation for the underlying pathophysiology. Interestingly, our patient unquestionably developed a rapid progressive cardiomyopathy following OLT, though with minimal change in overall myocardial thickness when compared to the preOLT echocardiogram. There was however a significant degree of change in left atrial volume. We postulate that the amyloid deposition may cause significant pathological changes on a cellular level leading to restriction, before there is macroscopic evidence of increasing ventricular thickness.

\section{Conclusion}

Therefore, the results to date indicate that paradoxical wild-type TTR deposition after OLT can preferentially occur in myocardium, leading to even fatal cardiac dysfunction. What are the long-term implications? Essentially, the long-term outcome after OLT in FAP is still unknown. Although malnutrition, neuropathy, and renal insufficiency may be ameliorated, there is concern that an accelerating restrictive cardiomyopathy may limit survival. FAP patients with Val30Met and especially those with nonVal30Met mutations must be followed by 
echocardiography on a regular basis after OLT to enable an identification of individuals with increasing cardiomyopathy. However, as was seen in our patient, an accelerated cardiomyopathy may present clinically before there is echocardiographic evidence of amyloid deposition. This finding should urge physicians to measure cardiac hemodynamics in this setting, especially when there is no significant change in ventricular thicknesss. Echocardiography with Doppler can be useful, but in our case, the diagnosis was made with cardiac catheterization. With more data, combined heart and liver transplantation might be considered as an initial management strategy, even in those with a non existent or a subclinical cardiomyopathy. Alternatively, a new medical trial such as stabilization of wild-type TTR and/or reduction of the amount of substrate available for amyloid formation using antisense oligonucleotides may in the future be therapeutic options for FAP patients, before and after OLT [8-10].

\section{Abbreviations}

1. FAP: Familial Amyloid Polyneuropathy

\section{OLT: Orthotropic Liver Transplantation}

3. TTR: Transthyretin

\section{ATTR: AmyloidgenicTTR}

\section{Competing interests}

The author(s) declare that they have no competing interests.

\section{Authors' contributions}

All authors were involved with the writing/reviewing of the manuscript. All authors approved the final manuscript.

\section{Consent}

Written informed consent was obtained from the patient for publication of this case report and any accompanying images. A copy of the written consent is available for review by the Editor-in-Chief of this journal.

\section{Acknowledgements}

Full written consent has been obtained by the patient for the submission of this manuscript for publication. Funding was neither sought nor obtained.

\section{References}

I. Shu-ichi lkeda, Masamitsu Nakazato, Yukio Ando, Gen Sobue: Familial transthyretin-type polyneuropathy in Japan: Clinical and genetic heterogeneity. Neurology 58(7): I00I-I007. April 9, 2002

2. Saraiva MJ: Sporadic Cases of Heriditary Systemic Amyloidosis. The New England Journal of Medicine 346(23): I I 8-I I 9.2002 June 6

3. [http://www.fapwtr.org]

4. Grazi G, Cescon M, Fabrizio S, Ercolani G, Ravaioli M, Arpessela G, Magelli C, Grigioni F, Cavallari A: Combined Heart and Liver Transplantation for Familial Amyloidotoc Neuropathy: Con- siderations From the Hepatic Point of View. Liver Transplantation 2003, 9(9):986-92.

5. Stangou A, Hawkins P, Heaton N, Rela M, Monaghan M, Nihoyannopoulos P, O'Grady J, Pepys M, Williams P: Progressive Cardiac Amyloidosis Following LiverTransplantation For Familial Amyloid Polyneuropathy: Implications for Amyloid Fibrillogenesis. Transplantation 66(2):229-233. July 27, 1998

6. Olofsson B, Backman C, Kmap K: Progression of cardiomyopathy after liver transplantation in patients with familial amyloidotic polyneuropathy, portuguese type. Transplantation 73(5):745-75I. March I5, 2002

7. Yazaki M, Tokuda T, Nakamura A, Higuchi K, Harihara Y, Baba S, Ikeda S: Cardiac Amyloid in Patients with Familial Amyloid Polyneuropathy Consists of Abundant Wild-Type Transthyretin. Biochem Biophys Res Commun 2000, 274:702-706.

8. Miller SR, Sekijima Y, Kelly JW: Native state stabilization by NSAIDs inhibits transthyretin amyloidogenesis from the most common familial disease variants. Lab Invest 2004, 84:545-552.

9. Sato T, Ando Y, Susuki S, Nakamura A, Mikami F, Kai H: Chromium (III) ion and thyroxine cooperate to stabilize the transthyretin tetramer and suppress in vitro amyloid fibril formation. FEBS Let 2006, 580:49|-496.

10. Benson M, Kluve-Beckerman B, Zeldenrust SR: Targeted suppression of an amyloidogenic transthyretin with antisense oligonucleotides. Muscle Nerve 2006, 33:609-618.

\section{Publish with Bio Med Central and every scientist can read your work free of charge}

"BioMed Central will be the most significant development for disseminating the results of biomedical research in our lifetime. "

Sir Paul Nurse, Cancer Research UK

Your research papers will be:

- available free of charge to the entire biomedical community

- peer reviewed and published immediately upon acceptance

- cited in PubMed and archived on PubMed Central

- yours - you keep the copyright

Submit your manuscript here:

http://www.biomedcentral.com/info/publishing_adv.asp 